\title{
Effectiveness of COVID-19 vaccines against SARS- CoV-2 infection with the Delta (B.1.617.2) variant: second interim results of a living systematic review and meta-analysis, 1 January to 25 August 2021
}

Thomas Harder $^{1}$, Wiebe Külper-Schiek ${ }^{1}$, Sarah Reda ${ }^{1}$, Marina Treskova-Schwarzbach ${ }^{1}$, Judith Koch ${ }^{1}$, Sabine Vygen-Bonnet ${ }^{1}$, Ole Wichmann ${ }^{1}$

1. Robert Koch Institute, Berlin, Germany

Correspondence: Thomas Harder (hardert@rki.de)

Citation style for this article:

Harder Thomas, Külper-Schiek Wiebe, Reda Sarah, Treskova-Schwarzbach Marina, Koch Judith, Vygen-Bonnet Sabine, Wichmann Ole. Effectiveness of COVID-19 vaccines against SARS-CoV-2 infection with the Delta (B.1.617.2) variant: second interim results of a living systematic review and meta-analysis, 1 January to 25 August 2021. Euro Surveill. 2021;26(41):pii=2100920. https://doi.org/10.2807/1560-7917.ES.2021.26.41.2100920

The Delta variant has become the dominant strain of SARS-CoV-2. We summarised the evidence on COVID19 vaccine effectiveness (VE) identified in 17 studies that investigated VE against different endpoints. Pooled VE was $63.1 \%$ (95\% confidence interval (CI): 40.9-76.9) against asymptomatic infection, $75.7 \%$ (95\% Cl: 69.3-80.8) against symptomatic infection and $90.9 \%(95 \% \mathrm{Cl}: 84.5-94.7)$ against hospitalisation. Compared with the Alpha variant, VE against mild outcomes was reduced by $10-20 \%$, but fully maintained against severe COVID-19.

The Delta variant (Phylogenetic Assignment of Named Global Outbreak (Pango) lineage designation B.1.617.2) of severe acute respiratory syndrome coronavirus 2 (SARS-CoV-2) was first reported in October 2020 and has spread to more than 180 countries globally [1]. Concerns were raised about how well the currently available vaccines protect against this variant. Since January 2021, the Robert Koch Institute (RKI), in collaboration with the National Immunisation Technical Advisory Groups (NITAGs) network coordinated by the European Centre for Disease Prevention and Control (ECDC) is performing a living systematic review on the efficacy, effectiveness and safety of coronavirus disease (COVID-19) vaccines authorised in the European Union (EU) (PROSPERO registration: CRD42020208935) [2]. Here we present results on the effectiveness and the duration of protection provided by the EU-licensed COVID-19 vaccines in respect to the Delta variant.

\section{Literature search}

This living systematic review follows the Preferred Reporting Items for Systematic Review and MetaAnalysis (PRISMA) guideline (Supplement Part S1). We considered studies of any design as long as they had a comparison group that investigated vaccine effectiveness (VE) against SARS-CoV-2 infection of any severity after vaccination with a COVID-19 vaccine (see Supplement Part S2 for complete population intervention comparison outcomes (PICO) question) approved by the European Medicines Agency. We did not apply any restrictions on publication language and status.

We searched the internal COVID-19 literature database constructed by the RKI library and hand-searched relevant websites (see [2] and Supplement Part S3 for description of the database and the search strategy). Potentially relevant publications were screened at title/abstract and full-text level by at least two out of three independent investigators (TH, WKS, SR). Disagreements on eligibility were resolved through discussion. Data were extracted from the included studies (see PROSPERO protocol for details) and summarised in tables. Only VE estimates of completed vaccination schedules were analysed. The risk of bias in non-randomized studies - of interventions (ROBINS-I) was used to assess risk of bias [3].

We performed meta-analyses, using a random-effects model to account for heterogeneity between studies. The $I^{2}$ was used to quantify the extent of heterogeneity. Formal testing for publication bias was done for datasets with 10 or more estimates by inspection of funnel plots, followed by Begg's test and Egger's test (Supplement Part S4).

\section{Study screening}

In total, 7,117 entries were identified and screened until 25 August 2021, the date of last search. Additionally, 11 potentially relevant studies were identified by 


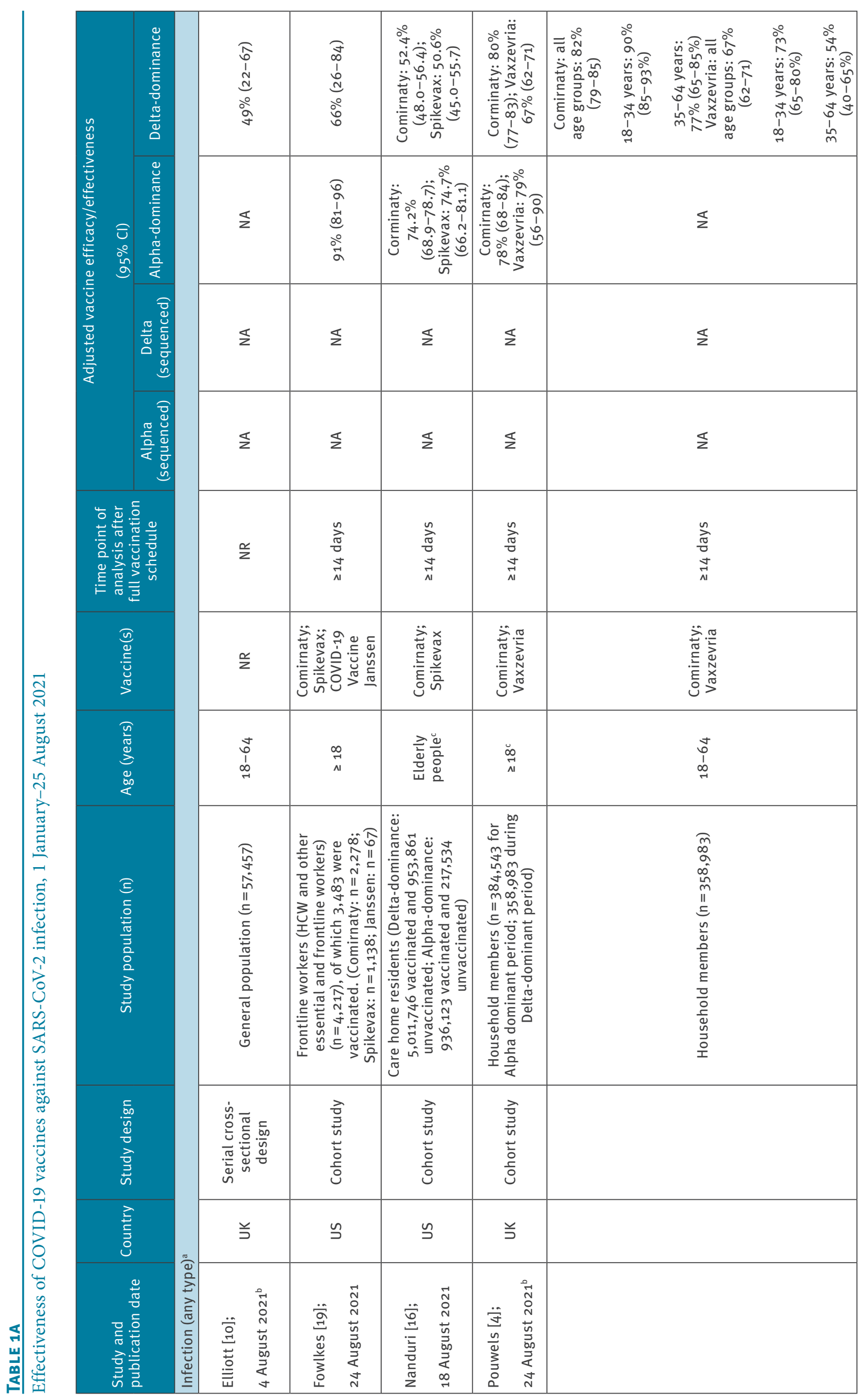



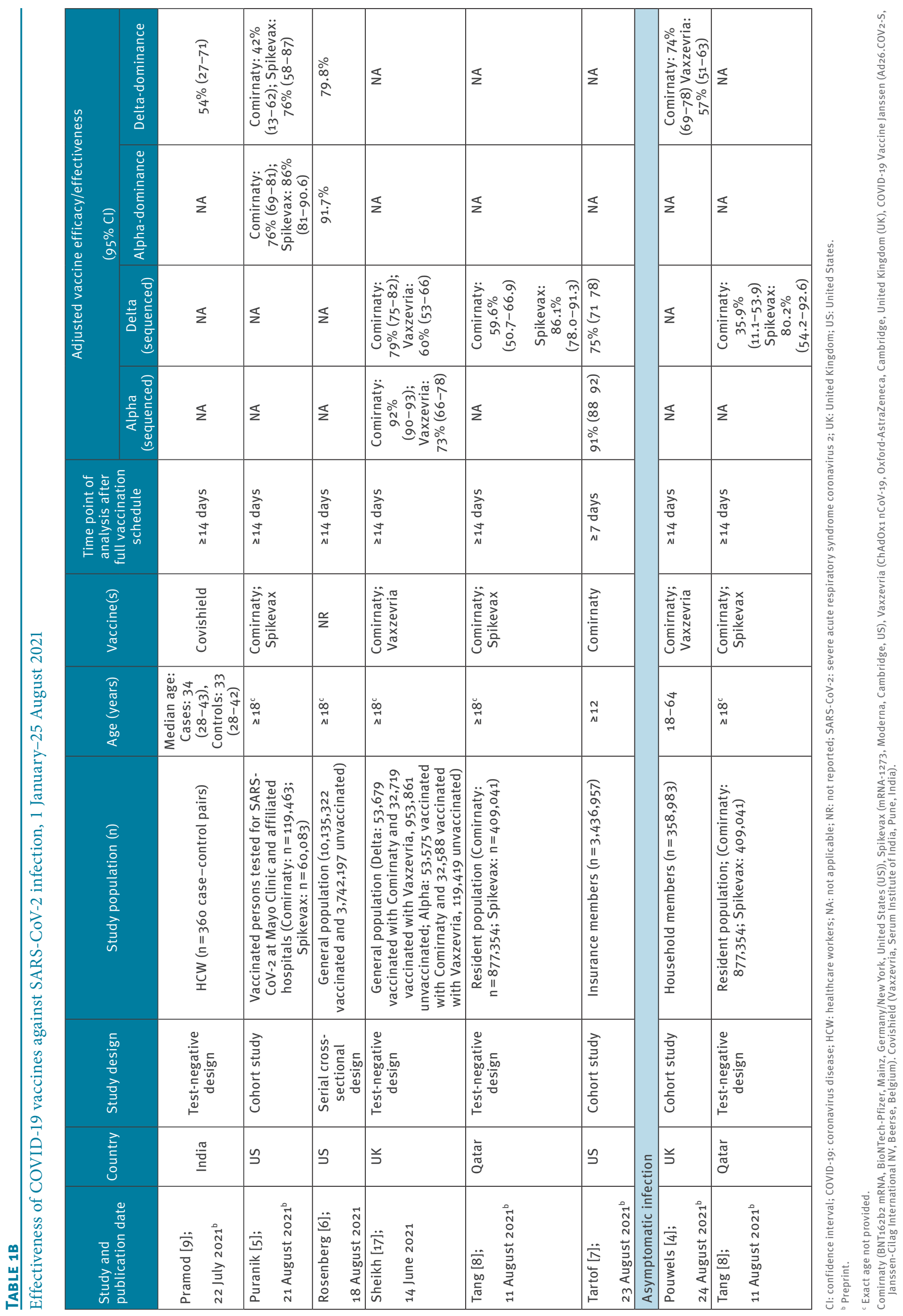


\section{FIGURE 1}

Results of the meta-analysis for SARS-CoV-2 infection outcomes, 1 January-25 August 2021

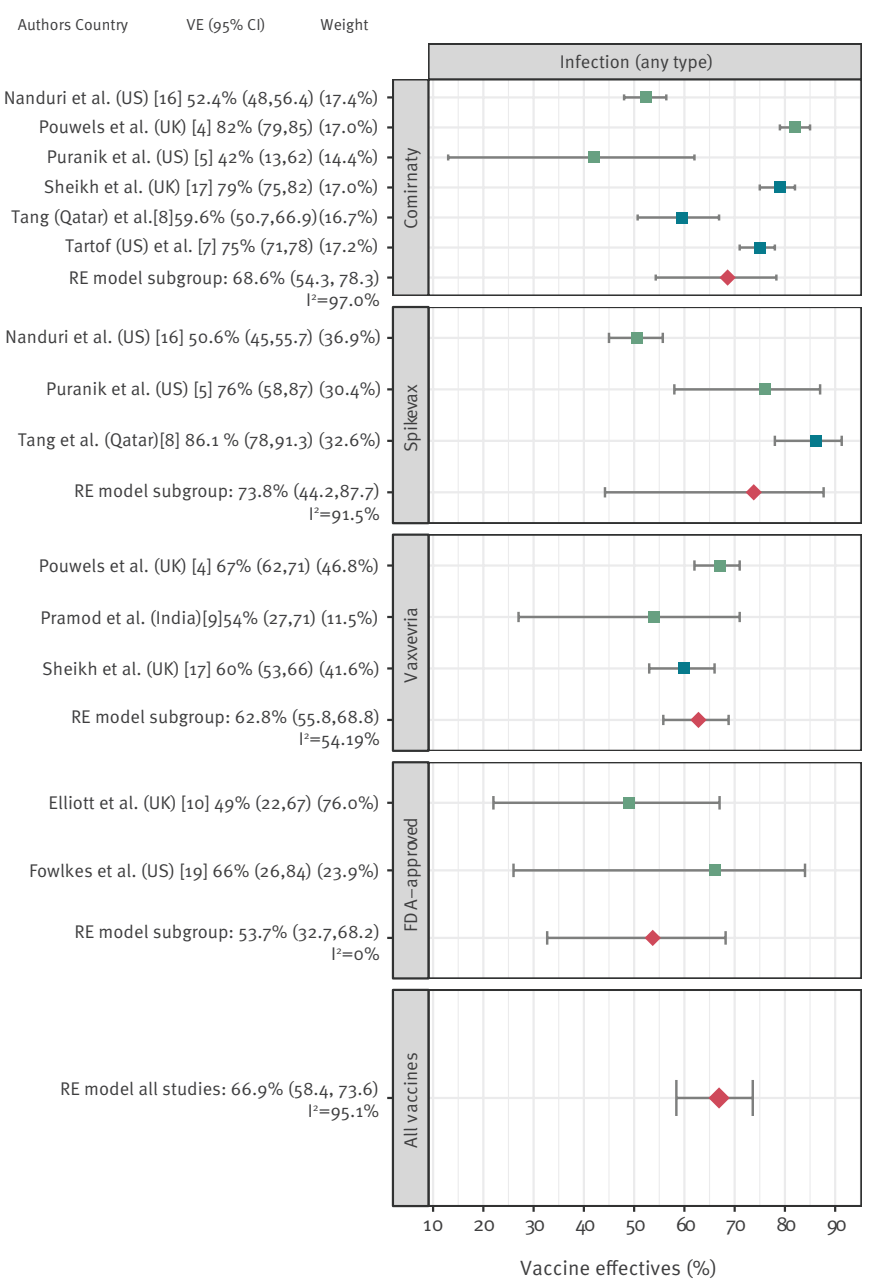

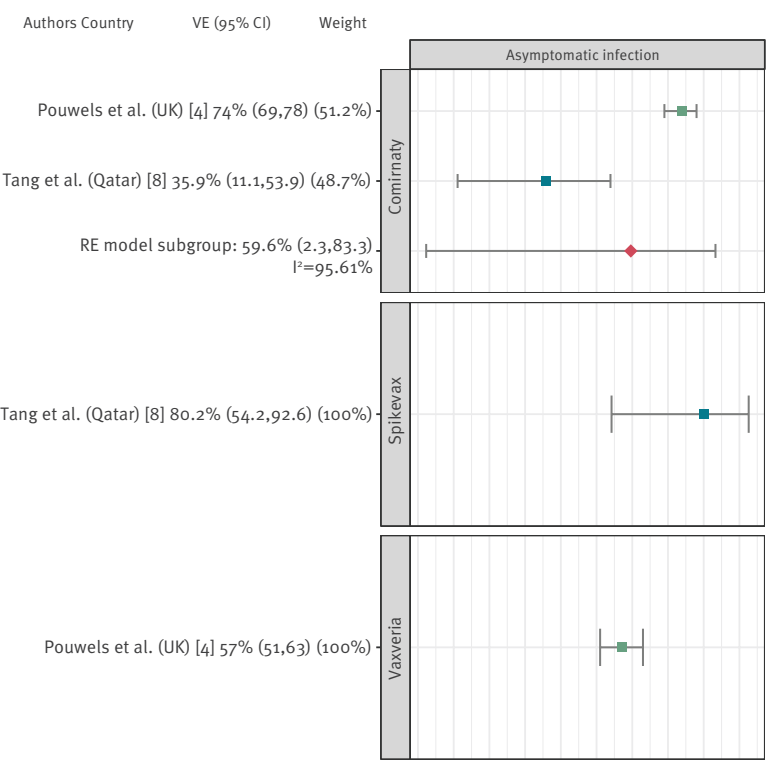

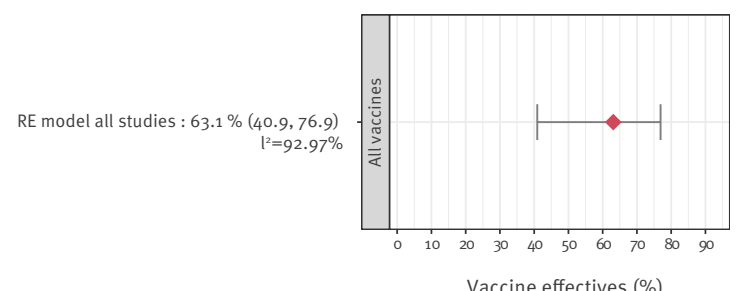

Vaccine effectives (\%)

FDA: The United States Food and Drug Administration; RE: Random effects; SARS-CoV-2: severe acute respiratory syndrome coronavirus 2; UK: United Kingdom; US: United States.

The y-axis illustrates study and reported VE estimates $(95 \% \mathrm{Cl}$ ) included in the meta-analysis (inverse variance weight for subgroup metaanalysis \%). The bottom box represents the results of the meta-analysis across all studies for the outcome (RE model all studies: Pooled VE\% (95\% CI) I-square statistic for all studies meta-analysis).

FDA-approved: Comirnaty (BNT162b2 mRNA, BioNTech-Pfizer, Mainz, Germany/New York, United States (US)), Spikevax (mRNA-1273, Moderna, Cambridge, US), COVID-19 Vaccine Janssen (Ad26.COV2-S, Janssen-Cilag International NV, Beerse, Belgium).

hand-searching. After full-text screening, 17 studies [4-20] were included (Supplement Part S5: PRISMA flowchart).

\section{Types of studies}

Included studies reported VE against infections with the SARS-CoV-2 Delta variant only, or compared VE against the Delta variant with estimates against the Alpha (B.1.1.7) variant. Within each of these study types, two methodological subtypes were identified: (i) studies that calculated VE against Delta and Alpha from sequenced samples; or (ii) studies that calculated
VE from time periods during which Delta and Alpha was the dominant strain in the respective study location without having sequenced each sample. Two studies $[7,19]$ also investigated VE at several time points after vaccination, thereby addressing waning vaccineinduced immunity.

\section{Prevention of any infection}

Of 17 studies, 10 [4-10,16,17,19] reported the effectiveness of COVID-19 vaccines in preventing SARS-CoV-2 infection but did not report whether these were symptomatic or asymptomatic infections; Table 1 ). Studies 


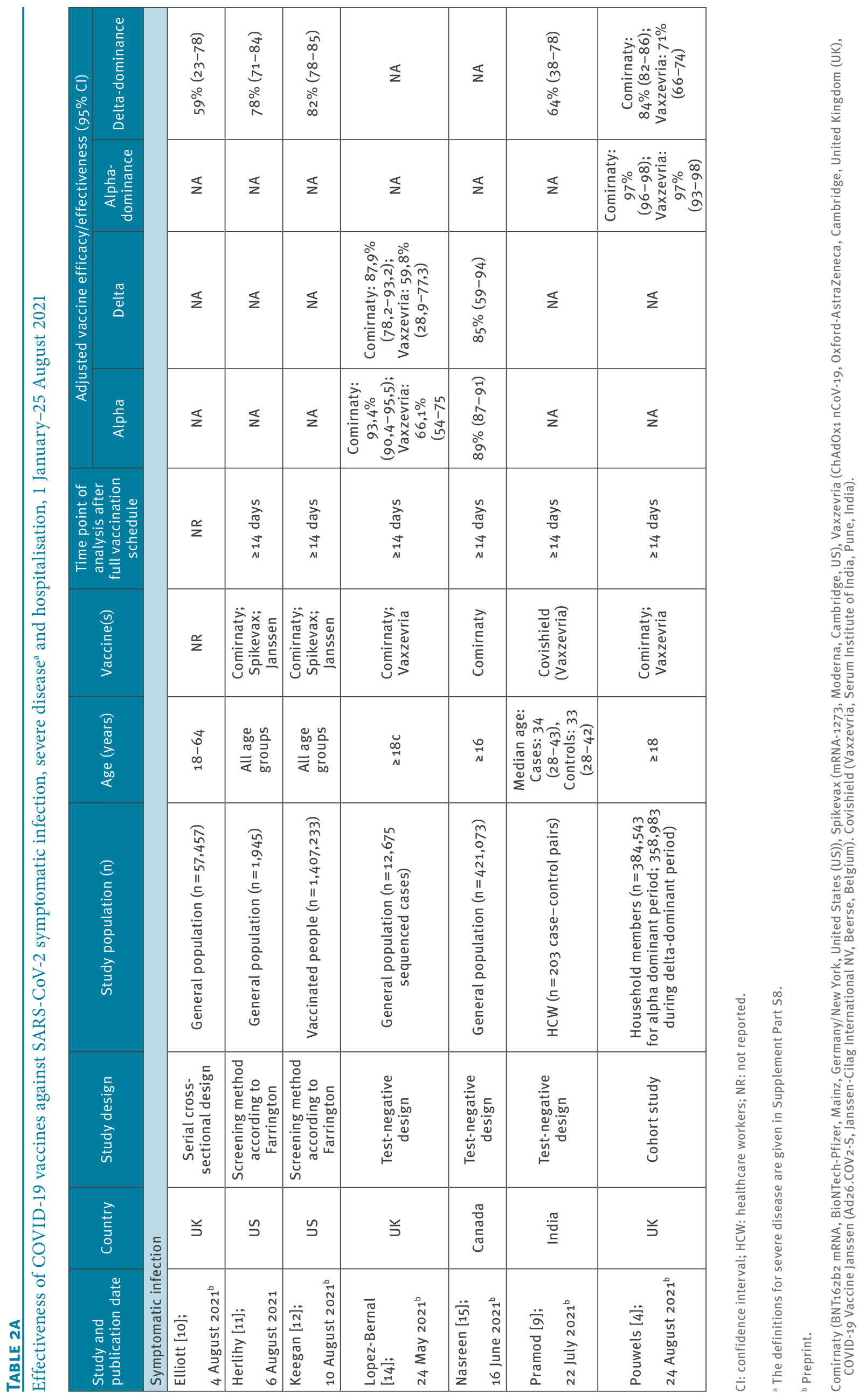



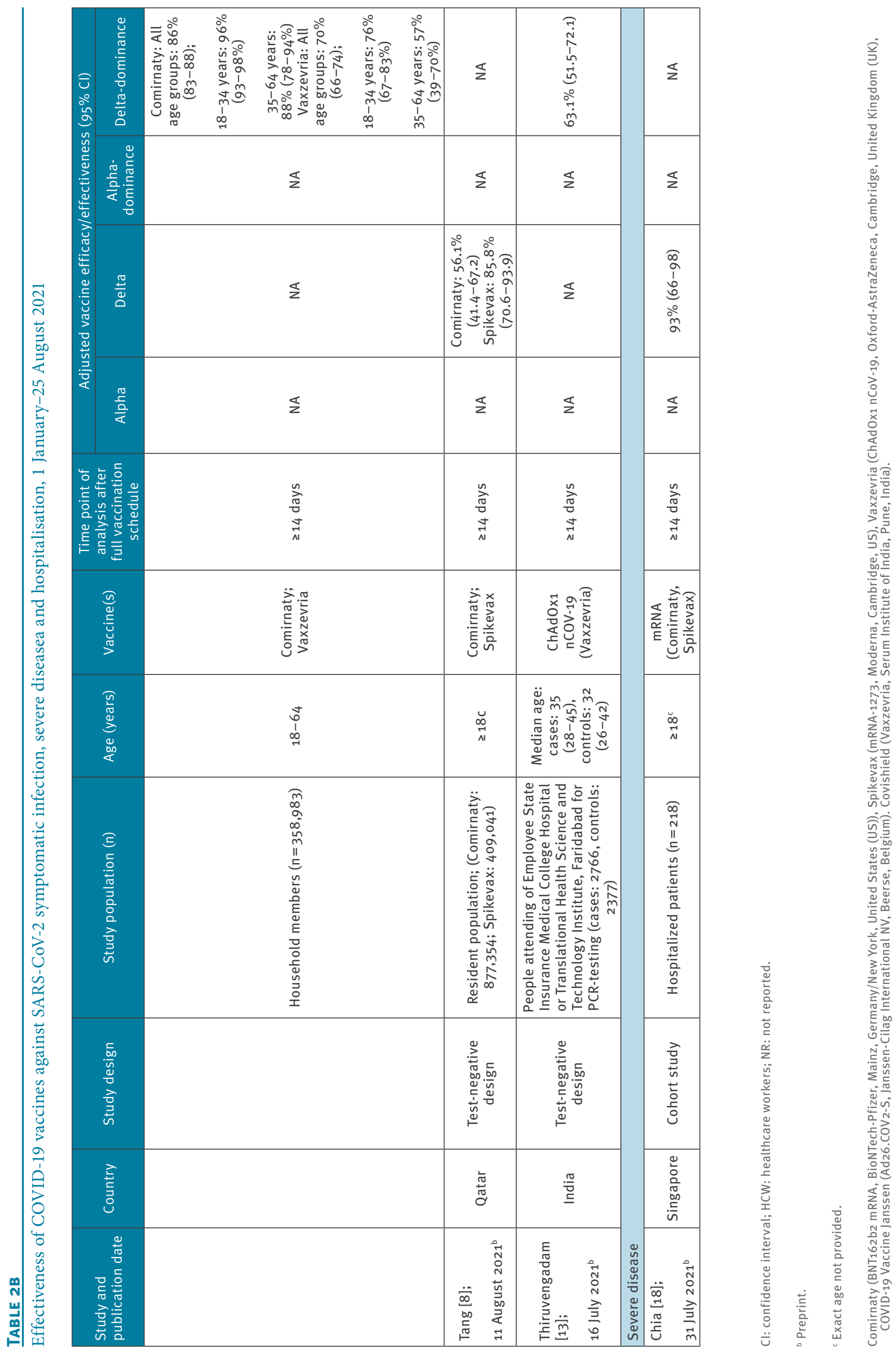


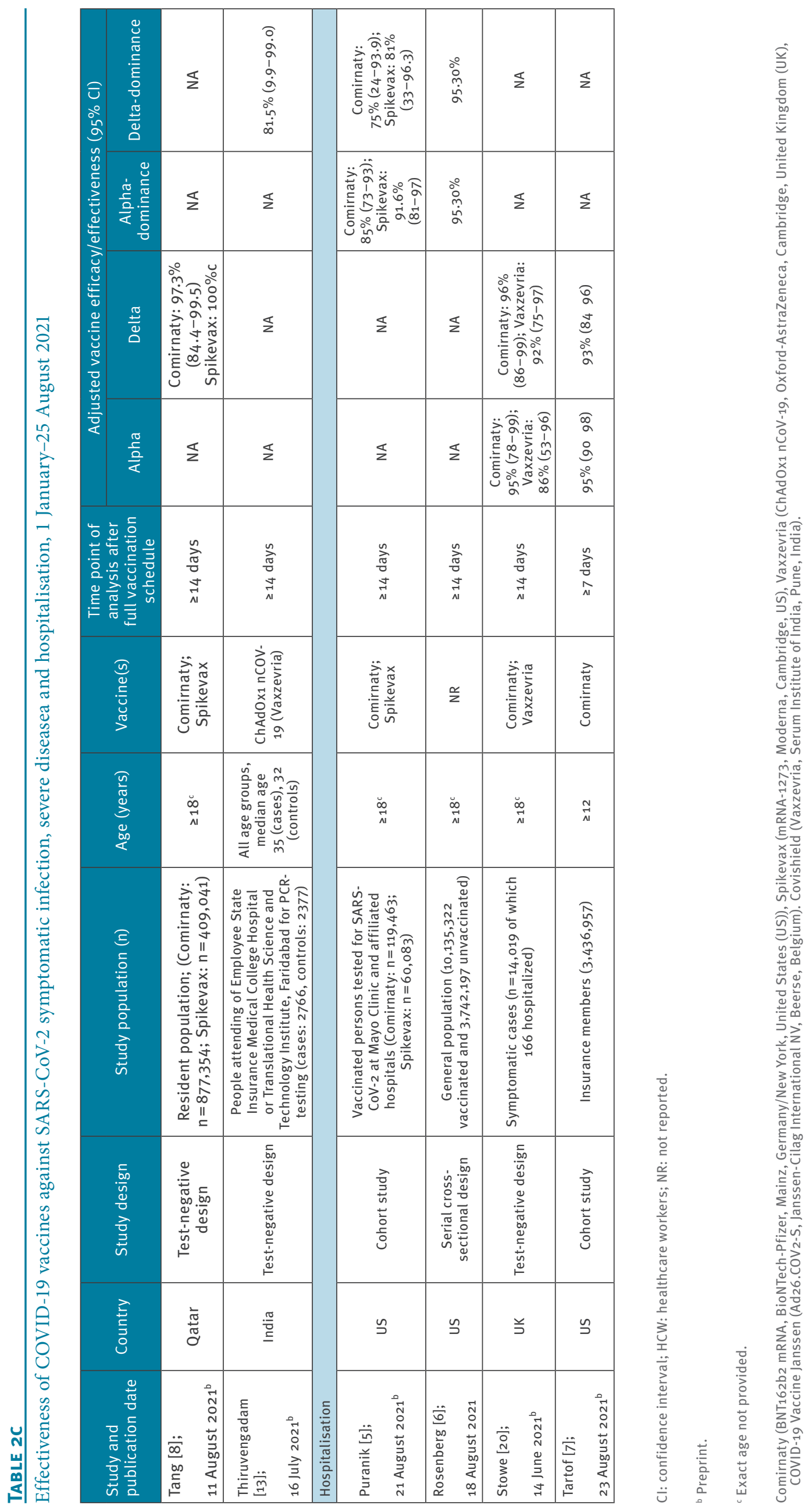


A

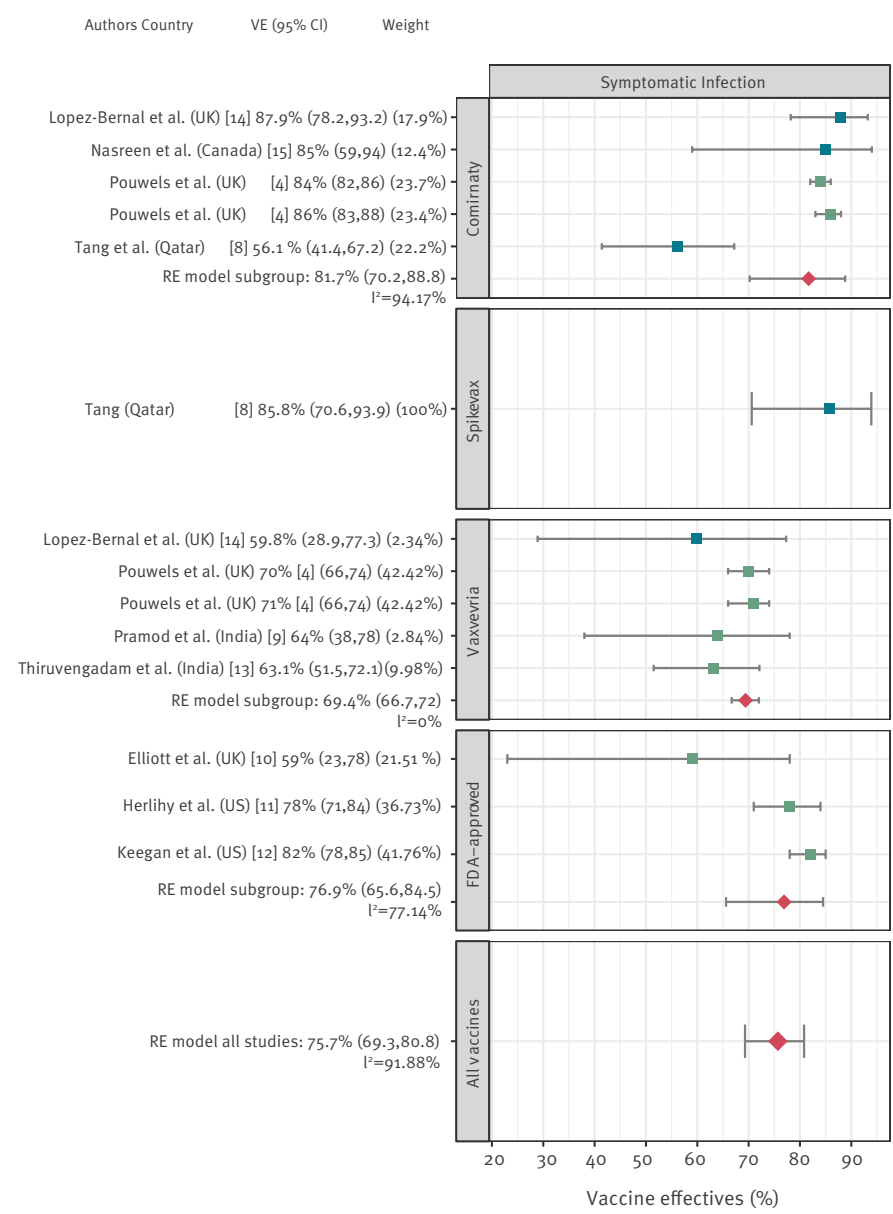

B.
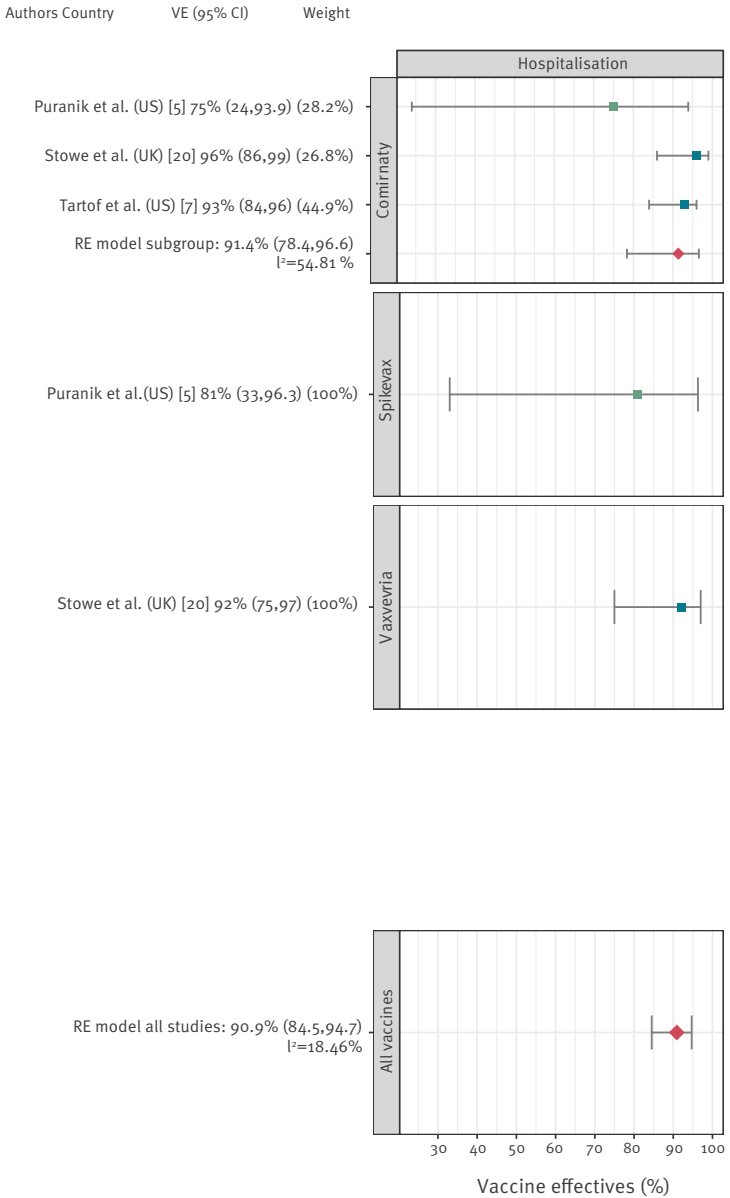

- Sequencing • Dominance $\bullet$ Meta-analysis estimate

$\mathrm{Cl}$ : confidence interval; FDA: The United States Food and Drug Administration; RE: Random effects; SARS-CoV-2: severe acute respiratory syndrome coronavirus 2; UK: United Kingdom; US: United States.

The y-axis illustrates study and reported VE estimates $(95 \% \mathrm{CI})$ included in the meta-analyses (inverse variance weight for subgroup metaanalysis \%). The bottom box represents the results of the meta-analysis across all studies for the outcome (RE model all studies: Pooled VE\% (95\% CI) I-square statistic for all studies meta-analysis).

were conducted in four countries. Five were cohort studies, three were test-negative case-control studies and two were based on serial cross-sectional samples. Six studies investigated more than one vaccine (Comirnaty (BNT162b2 mRNA, BioNTech-Pfizer, Mainz, Germany/New York, United States (US)) and Spikevax (mRNA-1273, Moderna, Cambridge, US) or Comirnaty and Vaxzevria (ChAdOx1 nCoV-19, Oxford-AstraZeneca, Cambridge, United Kingdom (UK) and COVID-19 Vaccine Janssen (Ad26.COV2-S, Janssen-Cilag International NV, Beerse, Belgium). One study each evaluated Comirnaty and Covishield (Vaxzevria, Serum Institute of India, Pune, India) and two studies did not specify the examined vaccines. The VE estimates in the included studies against any type of infection for all age groups, ranged between $49 \%$ and $82 \%$; in one study with information for $18-34$-year-olds the range was $90 \%$ [4]. Pooled VE was $66.9 \%$ (95\% confidence interval $(\mathrm{CI})$ : 58.4-73.6; $\mathrm{I}^{2}=95.1 \%$ ) across all studies (Figure $1 \mathrm{~A}$ ).

\section{Prevention of asymptomatic infection}

Two studies investigated VE against asymptomatic infections (Table 1). These studies were performed in the UK and Qatar $[4,8]$. Both studies investigated two vaccines. The VE estimates ranged between $35.9 \%$ and $80.2 \%$. Across studies, pooled VE was $63.1 \%(95 \% \mathrm{Cl}$ : 40.9-76.9; $\left.\right|^{2}=93 \%$; Figure $1 \mathrm{~B}$ ).

\section{Prevention of symptomatic infection}

Nine studies estimated the effectiveness of COVID-19 vaccines in preventing symptomatic SARS-CoV-2 infections (Table 2). Studies were performed in five countries [4,8-15]. Five studies had a test-negative design, two studies applied the screening method and one 
study each had a cohort design and used serial crosssectional samples. Six studies investigated more than one vaccine. The VE against symptomatic infection ranged between $56 \%$ und $87.9 \%$. The pooled VE estimate was $75.7 \%$ (95\% Cl: 69.3-80.8; $\mathrm{l}^{2}=91.9 \%$; Figure 2 A).

Prevention of severe disease and hospitalisation The VE against the compound outcome severe disease was assessed in three studies (one cohort, two testnegative design). They were performed in three countries (Table 2) $[8,13,18]$. The VE estimates ranged from $81.5 \%$ to $100 \%$ and the pooled VE was $93.8 \%$ (95\% Cl: 83-98; $1^{2}=0 \%$; Supplement Part S6). Hospitalisation was reported in four studies: two cohort studies, one test-negative design study and one analysis of cross-sectional serial samples (Table 2). The studies were performed in the US and the UK $[5-7,20]$. Single study VE ranged between $75 \%$ and $96 \%$. Pooled VE against hospitalisation was $90.9 \%$ ( $95 \% \mathrm{Cl}$ : 84.5-94.7; $\mathrm{I}^{2}=18.5 \%$; Figure $2 \mathrm{~B}$ ). No study reported admission to intensive care unit, intubation or death.

\section{Effectiveness against Delta variant compared with Alpha variant}

In nine studies, VE estimates against infections with the Delta variant were compared with those against infections with the Alpha variant in the same study for at least one outcome (Tables 1 and 2). Overall, VE against Delta was $10-20 \%$ lower than VE against Alpha for less severe outcomes. For hospitalisation, VE against Delta did not differ from VE against Alpha.

\section{Waning protection}

Two cohort studies from the US investigated VE against infections (symptomatic or asymptomatic) for more than one time point after vaccination. One study reported a decrease of protection offered by the Comirnaty vaccine from $93 \%(95 \% \mathrm{Cl}: 85-97)$ at baseline to $53 \%(95 \%$ $\mathrm{Cl}: 39-65)$ after at least 4 months [7]. The other study investigated protection conferred by any FDA-licensed vaccines in frontline workers (healthcare workers and others) and reported a non-significant change from $85 \%$ (95\% Cl: $68-93)$ to $73 \%$ (95\% Cl: $49-86)$ after at least 5 months following full vaccination [19].

\section{Risk of bias}

14 studies $[4,5,7-10,13-20]$ had a moderate risk of bias and three studies $[6,11,12]$ had a critical risk of bias. Major limitations were incomplete or absent adjustment for confounders (see Supplement Part S7 for details). No evidence of publication bias was detected.

\section{Discussion}

These second interim results of our living systematic review show that COVID-19 vaccines approved in the EU have a moderate to high effectiveness against mild to moderate forms of SARS-CoV-2 infections caused by the Delta variant, while VE against severe disease and hospitalisation was high to very high. Statistical heterogeneity was low in meta-analysis of the severe outcomes, further supporting a well-maintained effectiveness against these endpoints under Delta variant dominance.

In one study where VE was investigated in more than one age group, higher estimates in younger groups were seen. Of note, no VE estimate against Delta had been reported until data cut (25 August) for the Janssen vaccine.

As already discussed in the first interim analysis [2] and given the highly dynamic publishing landscape in this field, we cannot exclude the possibility that additional published studies were not captured by our search strategy. Further limitations stem from the fact that genomic sequencing was used to determine VE against Delta in only a minority of studies, while in the majority of studies VE was estimated during time periods of dominant Delta circulation without sequencing. Moreover, based on the current evidence, it is challenging to segregate two factors contributing to the difference between the VE estimates against Alpha and Delta variants: waning immunity and actual VE against Delta. Furthermore, a number of studies did not report separate estimates per vaccine.

\section{Conclusion}

Current evidence shows that COVID-19 vaccines licensed in the EU are moderately to highly effective in preventing SARS-CoV-2 infections with the Delta variant, while effectiveness against severe courses of COVID-19 remains high.

\section{Acknowledgements}

The authors would like to thank the members of the EU/EEA NITAG Collaboration Working Group 3 for valuable comments on the PICO questions and the study protocol.

\section{Conflict of interest}

None declared.

\section{Authors' contributions}

TH conceived the study performed study screening (as second reviewer) and drafted the manuscript. JK and SVB reviewed the results. SR and WKS performed study screening (as first reviewers) and contributed to the manuscript. MTS performed the meta-analysis. OW held general oversight of the work and revised the manuscript. All authors reviewed the manuscript, contributed to the interpretation of the data and provided intellectual input.

\section{References}

1. World Health Organization (WHO). Weekly epidemiological update on COVID-19 - 14 September 2021. Edition 57. Geneva: WHO; 2021. Available from: https://www.who.int/ publications/m/item/weekly-epidemiological-update-on-covid19---14-september-2021

2. Harder T, Koch J, Vygen-Bonnet S, Külper-Schiek W, Pilic A, Reda S, et al. Efficacy and effectiveness of COVID-19 vaccines against SARS-CoV-2 infection: interim results of a living. systematic review, 1 January to 14 May 2021. Euro Surveill. 
2021;26(28):2100563. https://doi.org/10.2807/1560-7917. ES.2021.26.28.2100563 PMID: 34269175

3. Sterne JA, Hernán MA, Reeves BC, Savović J, Berkman ND, Viswanathan M, et al. ROBINS-I: a tool for assessing risk of bias in non-randomised studies of interventions. BMJ. 2016;355:i4919. https://doi.org/10.1136/bmj.i4919 PMID: 27733354

4. Pouwels KB, Pritchard E, Matthews P, Stoesser NB, Eyre DW, Vihta K-D, et al. Impact of Delta on viral burden and vaccine effectiveness against new SARS-CoV-2 infections in the UK. medRxiv. 2021:2021.08.18.21262237V1. https://doi.org/10.110 $1 / 2021.08 .18 .21262237$

5. Puranik A, Lenehan PJ, Silvert E, Niesen MJ, Corchado-Garcia J, O'Horo JC, et al. Comparison of two highly-effective mRNA vaccines for COVID-19 during periods of Alpha and Delta variant prevalence. medRxiv. 2021:2021.08.06.21261707v3. https://doi.org/10.1101/2021.08.06.21261707

6. Rosenberg ES, Holtgrave DR, Dorabawila V, Conroy M, Greene D, Lutterloh E, et al. New COVID-19 cases and hospitalizations among adults, by vaccination status-New York, May 3-July 25, 2021. MMWR Morb Mortal Wkly Rep. 2021;70(34):1150-5. https://doi.org/10.15585/mmwr.mm7034e1 PMID: 34437517

7. Tartof SY, Slezak JM, Fischer H, Hong V, Ackerson BK, Ranasinghe ON, et al. Six-month effectiveness of BNT162B2 mRNA COVID-19 vaccine in a large US Integrated health system: a retrospective cohort study. The Lancet. 2021.

8. Tang P, Hasan MR, Chemaitelly H, Yassine HM, Benslimane F, Al Khatib HA, et al. BNT162b2 and mRNA-1273 COVID-19 vaccine effectiveness against the Delta (B. 1.617.2) variant in Qatar. medRxiv. 2021:2021.08.11.21261885V1. https://doi.org/10.1101 /2021.08.11.21261885

9. Pramod S, Govindan D, Ramasubramani P, Kar SS, Aggarwal $R$. Effectiveness of Covishield vaccine in preventing Covid-19-A test-negative case-control study. medRxiv. 2021:2021.07.19.21260693v1. https://doi.org/10.1101/2021.07 .19 .21260693

10. Elliott P, Haw D, Wang H, Eales O, Walters C, Ainslie K, et al. REACT-1 round 13 final report: exponential growth, high prevalence of SARS-CoV-2 and vaccine effectiveness associated with Delta variant in England during May to July 2021. Imperial College London: London; 2021. Available from: https://spiral.imperial.ac.uk/handle/10044/1/90800

11. Herlihy R, Bamberg W, Burakoff A, Alden N, Severson R, Bush E, et al. Rapid increase in circulation of the SARS-CoV-2 B.1.617.2 (Delta) variant - Mesa County, Colorado, April-June 2021. MMWR Morb Mortal Wkly Rep. 2021;70(32):1084-7. https://doi.org/10.15585/mmwr.mm7032e2 PMID: 34383734

12. Keegan L, Truelove SA, Lessler J. Progress of the Delta variant and erosion of vaccine effectiveness, a warning from Utah. medRxiv. 2021:2021.08.09.21261554V1. https://doi.org/10.110 $1 / 2021.08 .09 .21261554$

13. Thiruvengadam R, Awasthi A, Medigeshi G, Bhattacharya S, Mani S, Sivasubbu S, et al. Cellular immune responses are preserved and may contribute to Chadox1 ChAdOx1 NCoV-19 vaccine effectiveness against infection due to SARS-CoV-2 B.1.617.2 Delta variant despite reduced virus neutralisation. SSRN. 2021. Available from: https://papers.ssrn.com/sol3/ papers.cfm?abstract_id $=3884946$

14. Bernal JL, Andrews N, Gower C, Gallagher E, Simmons R, Thelwall S, et al. Effectiveness of COVID-19 vaccines against the B.1.617.2 variant. medRxiv. 2021:2021.05.22.21257658. https://doi.org/10.1101/2021.05.22.21257658

15. Nasreen S, He S, Chung H, Brown KA, Gubbay JB, Buchan SA, et al. Effectiveness of COVID-19 vaccines against variants of concern, Canada. medRxiv. 2021:2021.06.28.21259420v2. https://doi.org/10.1101/2021.06.28.21259420

16. Nanduri S, Pilishvili T, Derado G, Soe MM, Dollard P, Wu H, et al. Effectiveness of Pfizer-BioNTech and Moderna vaccines in preventing SARS-CoV-2 infection among nursing home residents before and during widespread circulation of the SARS-CoV-2 B. 1.617. 2 (Delta) Variant-National Healthcare Safety Network, March 1-August 1, 2021. MMWR Morb Mortal Wkly Rep. 2021;70(34):1163-6. https://doi.org/10.15585/ mmwr.mm7034e3 PMID: 34437519

17. Sheikh A, McMenamin J, Taylor B, Robertson C, Public Health Scotland and the EAVE II Collaborators. SARS-CoV-2 Delta VOC in Scotland: demographics, risk of hospital admission, and vaccine effectiveness. Lancet. 2021;397(10293):24612. https://doi.org/10.1016/S0140-6736(21)01358-1 PMID: 34139198

18. Chia PY, Xiang Ong SW, Chiew CI, Ang LW, Chavatte J-M, Mak T-M, et al. Virological and serological kinetics of SARS-CoV-2 Delta variant vaccine-breakthrough infections: a multi-center cohort study. medRxiv. 2021:2021.07.28.21261295. https:// doi.org/10.1101/2021.07.28.21261295
19. Fowlkes A, Gaglani M, Groover K, Thiese MS, Tyner H, Ellingson K, HEROES-RECOVER Cohorts. Effectiveness of COVID-19 Vaccines in Preventing SARS-CoV-2 Infection Among Frontline Workers Before and During B.1.617.2 (Delta) Variant Predominance - Eight U.S. Locations, December 2020-August 2021. MMWR Morb Mortal Wkly Rep. 2021;70(34):1167-9. https://doi.org/10.15585/mmwr.mm7034e4 PMID: 34437521

20. Stowe J, Andrews N, Gower C, Gallagher E, Utsi L, Simmons R. Effectiveness of COVID-19 vaccines against hospital admission with the Delta (B. 1.617. 2) variant. Public Health England. 2021. Available from: https://media.tghn.org/articles/ Effectiveness_of_COVID-19_vaccines_against_hospital_ admission_with_the_Delta_B._G6gnnqJ.pdf

\section{License, supplementary material and copyright}

This is an open-access article distributed under the terms of the Creative Commons Attribution (CC BY 4.0) Licence. You may share and adapt the material, but must give appropriate credit to the source, provide a link to the licence and indicate if changes were made.

Any supplementary material referenced in the article can be found in the online version.

This article is copyright of the authors or their affiliated institutions, 2021. 